\title{
SCIDiC
}

\author{
International Journal of Dentistry and Oral Science (IJDOS) \\ ISSN: 2377-8075
}

\section{Assessment Of Oral Submucous Fibrosis Using Ultrasound As An Adjunct To Clinical Evaluation}

Research Article

\author{
Dr. Krishna S. Kumar ${ }^{1 *}$, Dr. Jayaprasad Anekar², Dr. Raj Achikanam Chirakara ${ }^{3}$
}

${ }^{1}$ Assistant Professor, Amrita School of Dentistry (Amrita Vishwavidyapeetham), AIMS Health care campus, Edappily, Kochi, Kerala(Current Affliation), Post graduate student, Department of Oral Medicine and Radiology, KVG Dental College and Hospital, Sullia DK, Karnataka, India. (Affliation where the work was primarily carried out).

${ }^{2}$ Professor and Head, KVG Dental College and Hospital, Sullia DK, Karnataka, India.

${ }^{3}$ Professor and Head, MAHE Institute of Dental Sciences and Hospital, Chalakkara, Pallor, Mahe, Puducherry, India.

\section{Abstract}

Objectives: To Evaluate oral tissues affected by Oral submucous fibrosis using Ultrasound Color Doppler. Comparative evaluation of the clinical stages of Oral submucous fibrosiswith Ultrasound Color Doppler findings will be done thereby assessing the severity of the disorder.

Methods and Material: The study included 39 case subjects, who were grouped into 3 stages based on clinical characteristics. Control group had 40 healthy subjects from the same geographic population who were both age and sex matched. Both the case and control group were subjected to ultrasound evaluation of buccal mucosa using a medical sonographic unit GE VOLUSON 730 pro, Color Doppler Ultrasound system.Statisticalanalysis was done using ANOVA test and fisher's exact test. Results: In the control group all subjects demonstrated a hypoechoic submucosa which could be clearly differentiated from the muscle layer. In the case group there was increased echogenicity compared to the control group. Submucosal thickness increased and vascularity was decreased as the disease progressed to stage II and stage III. In advanced cases of oral submucous fibrosis submucosa could not be differentiated from the muscle layer clearly.

Conclusions: Ultrasound Color Doppler examination can be a promising diagnostic tool for oral submucous fibrosis. It can even pick up positive sites affected by the disease in initial stages which may not be evident in clinical examination alone and hence can be used as an adjunctive investigation modality and for assessment of the severity of the disease, especiallywhere doing biopsy is not feasible.

Keywords: Buccal Mucosa; Color Doppler; Oral Submucous Fibrosis; Ultrasound.

\section{Introduction}

Oral submucous fibrosis (OSMF) is a common potentially (pre) malignant lesion [1] affecting the oral mucosa which might extend even into the pharynx, oesophagus or rarely into the larynx. It is commonly seen affecting individuals of South Asian countries especially among the Indian population where chewing of betel quid with arecanut is a customary practice. Increasing use of commercially manufactured products of betel quid which contain tobacco, arecanut and other substances in powdered or granulated form is also extremely alarming as it is associated with increased risk of malignancy [2].
Clinically OSMF is characterized bypresence of fibrous bands, blanching of mucosa and burning sensation especially on consumption of spicy foods. Reduction inmouth opening and cheek flexibility along with difficulty in tongue movements will be seen as a result of the fibrosis of the oral tissues [3, 4]. Involvement of the underlying submucosa and muscles may be present with disease progression. There will be also obliteration of blood vessels resulting in decreased vascularity and epithelial atrophy.Clinical assessment is subjective due to observer variability and alone it may be insufficient to assess the disease severity. Disease severity will be different in different sites of the oral cavity and biopsy

*Corresponding Author:

Dr. Krishna S. Kumar MDS,

Assistant Professor, Amrita School of Dentistry (Amrita Vishwavidyapeetham), AIMS Health care campus, Edappily, Kochi, Kerala(Current Affliation), Post graduate student,

Department of Oral Medicine and Radiology, KVG Dental College and Hospital, Sullia DK, Karnataka, India. (Affliation where the work was primarily carried out).

Tel: 8330875475

E-mail: krishna.santhosh32@gmail.com

Received: September 19, 2021

Accepted: November 17, 2021

Published: November 20, 202

Citation: Dr. Krishna S. Kumar, Dr. Jayaprasad Anekar, Dr. Raj Achikanam Chirakara. Assessment Of Oral Submucous Fibrosis Using Ultrasound As An Adjunct To Clinical Evaluation. Int J Dentistry Oral Sci. 2021;8(11):5042-5048. doi: http://dx.doi.org/10.19070/2377-8075-210001016

Copyright: Dr. Krishna S. Kumar MDS ${ }^{\circ} 2021$. This is an open-access article distributed under the terms of the Creative Commons Attribution License, which permits unrestricted use, distribution and reproduction in any medium, provided the original author and source are credited. 
taken from a single site may not characterize the severity of the entire disease. Hence the correlation between clinical and histological grading of the disease is poor [2].

Ultrasound (US) examination is a real time imaging modality which can provide both qualitative and quantitative assessment of the disease [3]. Color Dopplerprovides color coded representation of the perfused areas and gives information of vascularity of different tissues [5]. Hence this study aims in the assessment of the severity of OSMF using ultrasound and Color Doppler and the comparative evaluation of the stages of OSMF with ultrasound and color Doppler examination findings.

\section{Materials and Methods}

The study group consisted of 79 patients who visited the Department of Oral Medicine and Radiology in a teaching dental hospital, Sullia.

Patients were divided in to case and control groups (age and sex matched). An ethical clearance certificate was obtained from the institutional ethical committee to proceed with the study. Each sample of the selected group was informed about the study in patients known language and informed consent was obtained. The control group (Group -1) consisted of 40 patients with a negative history of chewing arecanut or its commercial preparations. Study group (Group-2) consisted of 39 Patients who were clinically diagnosed suffering from various stages of OSMF.

\section{The exclusion criteria were:}

Patients with known history of:

- Trismus owing to causes other than OSMF

- Previous biopsy or treatment for OSMF

- Any mucosal conditions where blood flow of the oral tissues are affected which includes anaemia, oral cancer and other coexisting oral mucosal lesions.

- Any systemic and skin diseases which includes scleroderma, amyloidosis, diabetes mellitus and hypertension.

- Radiation therapy done in the head and neck region

Both the case and control group were subjected to sonographic evaluation of buccal mucosa in the Department of Radiology, KVG Medical College.

Detailed history was elicited from each subject of group-2 and the data was entered into a structured Performa. The clinical examination was carried out and patients were divided into 3 stages with 13 patients in each group according to the classification proposedby Bose and Balan [6].

Two reference points were considered for ultrasonographic imaging of buccal mucosa. The first reference point indicated was posterior buccal mucosa (PBM) which was $1 \mathrm{~cm}$ anterior to the anterior border of the masseter muscle. The second reference point was anterior buccal mucosa $(\mathrm{ABM})$ which was $1 \mathrm{~cm}$ posterior to the commissure of the lip. An imaginary line was drawn between ABM and PBM [7] (Figure 1). Palpation for the presence

Figure 1. Facial photograph showing points marked for imaging for imaging of buccal mucosa. The first point was $1 \mathrm{~cm}$ anterior to the anterior border of the masseter muscle, indicating the posterior buccal mucosa (PBM) and the second was 1 $\mathrm{cm}$ posterior to the commissure of the lip indicating the anterior buccal mucosa (ABM).

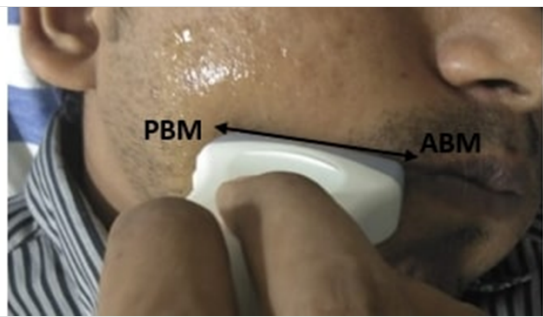

Figure 2. Position of the transducer is along the line joining the angle of mouth to the inferior point of the tragus of ear parallel to the lower border of the mandible.

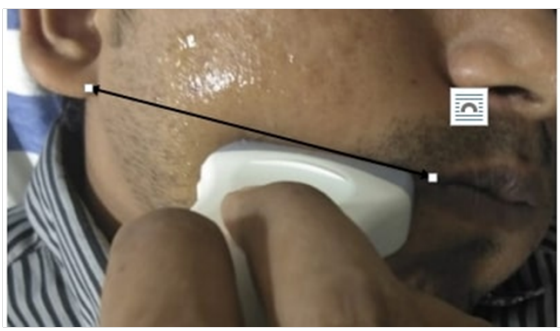

Figure 3. For imaging of the anterior buccal mucosa, anterior end of the probe was placed at the angle of the mouth with patient asked to blow the cheek.

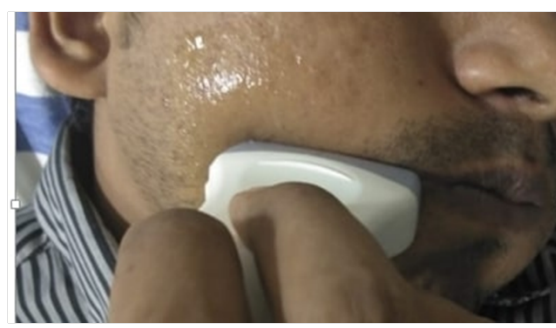


of fibrous bands was done in; Left anterior buccal mucosa (LAB), Left posterior buccal mucosa (LPB), Right anterior buccal mucosa (RAB), Right posterior buccal mucosa (RPB), for comparative evaluation with the ultrasound findings [2].

\section{Ultrasound evaluation}

Ultrasound scan of the buccal mucosa bilaterally were performed for all subjects using a medical sonographic unit GE VOLUSON 730 pro, Color Doppler Ultrasound system. A multifrequency linear transducer with frequency range from $6-13 \mathrm{MHz}$ was used. To avoid any bias related to the assessment of the echogenicity of the tissues technical parameters of the scan were maintained same during the entire study. Patient was made to lie in the supine position for the scan and it was carried out for anterior and posterior buccal mucosa separately on each side.

For imaging of the buccal mucosa transcutaneous extraoral transducer was positioned onthe cheek along an imaginary line which joins the inferior point of the tragus of the ear to the commissure of the mouth. This line will be parallel to the lower border of the mandible (Figure 2). Patient was asked to blow the cheek so as to define the mucosal structures clearly [8]. For imaging of the anterior buccal mucosa, anterior end of the probe was placed 1 $\mathrm{cm}$ posterior to the commissure of the lip (Figure 3). For the posterior buccal mucosa, the posterior end of the probe was placed
$1 \mathrm{~cm}$ anterior tothe anterior border of masseter muscle (Figure 4). US findings were recorded separately for the right and left anterior and posterior buccal mucosa. The echotexture of submucosa and its differentiation from the underlying muscle layer was recorded. The blood flow of the buccal mucosa was noted using Color Doppler Ultrasound.

Irrespective of the study findings all the patients included in this study underwent routine management for OSMF.

\section{Results}

The US appearance of the cross section of buccal mucosa in the control group was as follows. The oral mucosal lining appeared as a hyperechoic line (black arrow). Submucosa (SM) corresponds to the hypoechoic zone supported by an echogenic muscle layer corresponding to the buccinator muscle (BML). Submucosa could be clearly differentiated from the muscle layer. Buccal pad of fat (BPF) appears as a hypoechoic zone and muscles of facial expression (SFM) can be also appreciated (Figure 5). This US appearance of the layers of cheek in controls were similar to the normal echo architecture of the cheek layers from skin to the mucosa as reported in previous studies [2].

The obtained values of this study were subjected to statistical analysis using SPSS software version 20. One way ANOVA TEST

Figure 4. For the posterior buccal mucosa, the posterior end of the probe was placed at the anterior border of masseter muscle with patient asked to blow the cheek.

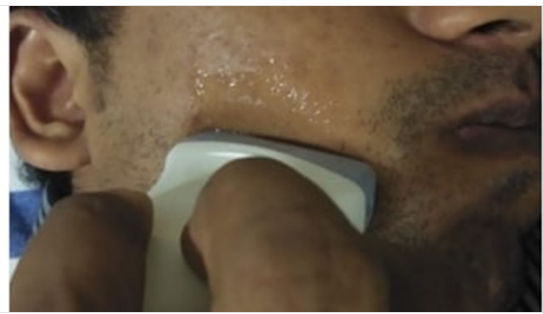

Figure 5. Normal sonographic appearance of the buccal mucosa in the control group the layers of the cheek are seen. They are (from below upwards) the hyperechoic oral mucosal lining (black arrow), the hypoechoic submucosa (SM), the echogenic buccinator muscle layer (BML), the hypoechoic buccal pad of fat (BPF) the superficial muscles of facial expression

(SFM). Distinct differentiation between the submucosa and the muscle layer is seen.

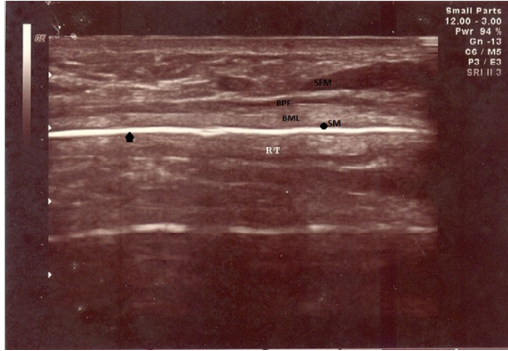

Figure 6. Increased echogencity of submucosa (white arrow) in OSMF stage 1 cases. Echo differentiation between the two layers is also indistinct.

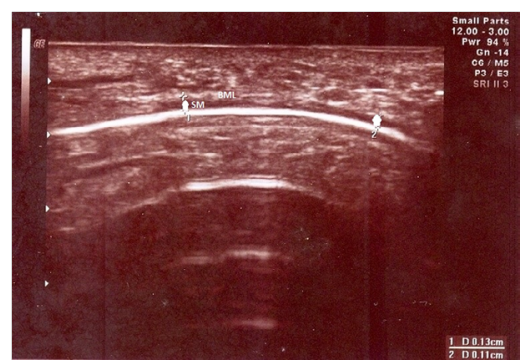


Figure 7. Hyperchoic submucosa (white arrow) in OSMF stage 3 case. There is marked echogenicity of the submucosa to approximate that of the muscle layer (MU) and the total loss of echo differentiation between the two layers. Significant increase in the thickness of the submucosa can also be appreciated.

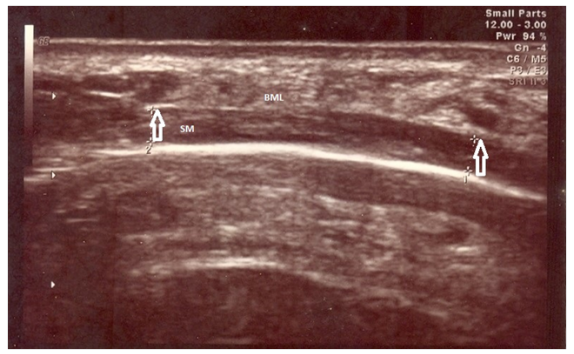

Figure 8. Color Doppler examination reveals normal vascularity of the buccal mucosa.

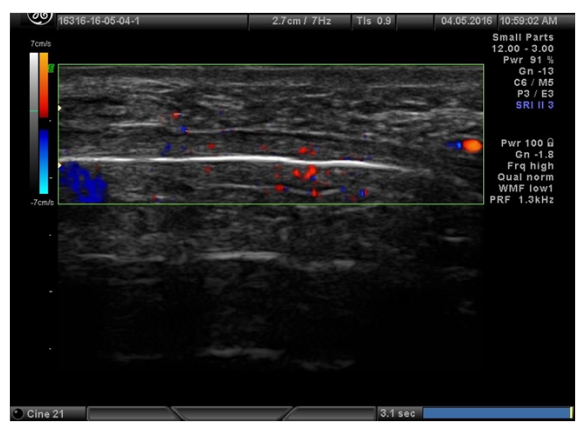

Figure 9. Color Doppler examination reveals decreased vascularity of the buccal mucosa in a stage 3 OSMF case.

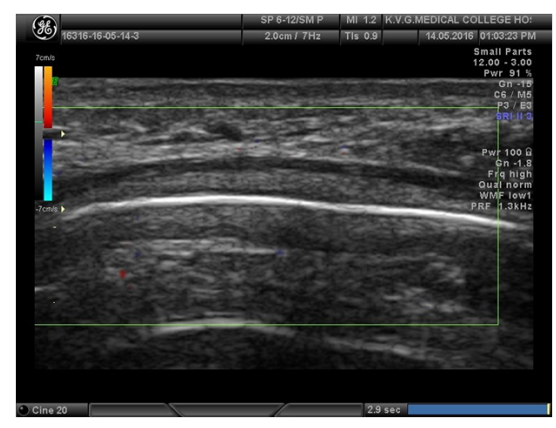

was used for the comparison of values of the case and control group. The threshold for significance was set at $\mathrm{p}<0.05$. Out of 79 subjects, 66(83.5\%) were males and $13(16.4 \%)$ were females. Mean age of patients in group- 1 was 32 years and group- 2 was 31.7 years. Comparison of AGE using one way ANOVA test showed that there was no statistical significance for the difference in age between the control and case group. Comparison of groups based on gender showed that maximum patients in all the case groups were males. There were 4 females $(30.8 \%)$ and 9 males $(69.2 \%)$ in stage 1 group, 2 females $(15.4 \%)$ and 11 males $(84.6 \%)$ in stage 2 group and 1 female (7.7\%) and 12 males (92.3) in stage 3 group. (Table 1 )

Comparison of case group and controls with the number of fibrous bands present clinically and with the number of sites positive in ultrasound examination showed that maximum number of fibrous bands and sites positive was present in stage 3 OSMF cases and minimum in control cases. Comparison of echotexture seen in various groups showed that control group had hypoechoic echotexture whereas in case group it appeared hyperechoic for stage 2 and stage 3 . In stage 1 case group, 10 patients $(76.9 \%)$ had hyperechoic areas and 3 patients $(23.1 \%)$ had hypoechoic areas. In the case group, all stage 2 and stage 3 cases showed hyperechoic appearance. Clear differentiation between the submucosa and the muscle layer was found in all patients of the control group. In the case group it was not distinct and as the disease progressed it was totally lost. In Stage 1 group of patients, in 10 cases $(76.9 \%)$ differentiation was distinct and in 3 cases $(23.1 \%)$ it was poor. In stage 2 and stage 3 group of patients there was total loss of differentiation. (Table 2, Figure 6 and Figure 7).

The mean submucosal thickness of controls was found to be $0.48 \mathrm{~mm}$. Submucosal thickness increased as the disease progressed from stage 1 to stage 3 in the case group. (Figure 6 and figure 7) In 2(15.4\%) stage 1 cases, thickness of the submucosa fell under the normal range while 7 cases $(53.8 \%)$ had mild increase and $4(30.8 \%)$ cases had increased thickness of submucosa. Increase in the thickness of the submucosa was found to be more in the posterior buccal mucosa compared to the anterior buccal mucosa on both right and left side. (Table 1 and Graph 1)Post Hoc Tukey analysis was done and it showed statisticallysignificant difference between all the pairs ( $p$ value $<0.05)$ except between the control and grade 1 group ( $\mathrm{p}>0.05)$.

On Color Doppler examination all group 1 controls (100\%) demonstrated normal vascularity of buccal mucosa.(Figure 8) In group 2, stage 1 cases $(100 \%)$ demonstrated normal vascularity. In stage 2 group, 8 cases $(61.5 \%)$ showed decreased vascularity and 5 cases showed normal vascularity, whereas all stage 3 OSMF patients $(100 \%)$ demonstrated decreased vascularity. (Figure 9) This shows that there is decrease in vascularity as the disease progresses to stage 3.(Table 2) 
Table 1. One way ANOVA test for comparison of the measurements.

\begin{tabular}{|c|c|c|c|c|}
\hline & & $\mathbf{N}$ & Mean + Std. Deviation & $\mathrm{p}$ value \\
\hline \multirow{5}{*}{ AGE } & Control & 40 & $32+5.7$ & \multirow{5}{*}{0.869} \\
\hline & Stage i & 13 & $30.69+7$ & \\
\hline & Stage ii & 13 & $31.85+4.8$ & \\
\hline & Stage iii & 13 & $32.62+6.9$ & \\
\hline & Total & 79 & $31.86+5.9$ & \\
\hline \multirow{5}{*}{$\begin{array}{l}\text { Anterior buccal } \\
\text { mucosa-R }\end{array}$} & Control & 40 & $0.4875+.07$ & \multirow{5}{*}{$<0.001$} \\
\hline & Stage i & 13 & $0.630769+.09$ & \\
\hline & Stage ii & 13 & $1.253846+0.6$ & \\
\hline & Stage iii & 13 & $1.846154+0.3$ & \\
\hline & Total & 79 & $0.860759+.5$ & \\
\hline \multirow{5}{*}{$\begin{array}{c}\text { Anterior buccal } \\
\text { mucosa-L }\end{array}$} & Control & 40 & $0.485+.07$ & \multirow{5}{*}{$<0.001$} \\
\hline & Stage i & 13 & $0.646154+0.2$ & \\
\hline & Stage ii & 13 & $1.353846+0.7$ & \\
\hline & Stage iii & 13 & $1.992308+0.2$ & \\
\hline & Total & 79 & $0.902532+0.6$ & \\
\hline \multirow{5}{*}{$\begin{array}{c}\text { Posterior buccal } \\
\text { mucosa-R }\end{array}$} & Control & 40 & $0.475+.07$ & \multirow{5}{*}{$<0.001$} \\
\hline & Stage i & 13 & $0.661538+0.1$ & \\
\hline & Stage ii & 13 & $1.546154+0.8$ & \\
\hline & Stage iii & 13 & $2.076923+0.4$ & \\
\hline & Total & 79 & $0.94557+0.7$ & \\
\hline \multirow{5}{*}{$\begin{array}{l}\text { Posterior buccal } \\
\text { mucosa-L }\end{array}$} & Control & 40 & $0.47+.06$ & \multirow{5}{*}{$<0.001$} \\
\hline & Stage i & 13 & $0.730769+.24$ & \\
\hline & Stage ii & 13 & $1.430769+.62$ & \\
\hline & Stage iii & 13 & $2.261538+0.4$ & \\
\hline & Total & 79 & $0.965823+0.7$ & \\
\hline
\end{tabular}

Table 2. Fishers exact test comparing the various categorical parameters with stages of OSMF and control.

\begin{tabular}{|c|c|c|c|c|c|c|}
\hline & & \multicolumn{4}{|c|}{ Groups } & \multirow{2}{*}{$\mathrm{p}$ value } \\
\hline & & Controls & Stage I & Stage II & Stage III & \\
\hline \multirow{5}{*}{$\begin{array}{l}\text { Fibrous bands present clin- } \\
\text { ically }\end{array}$} & 0 & 40 & 13 & 0 & 0 & \multirow{5}{*}{$<0.001$} \\
\hline & 1 & 0 & 0 & 1 & 0 & \\
\hline & 2 & 0 & 0 & 10 & 0 & \\
\hline & 3 & 0 & 0 & 1 & 0 & \\
\hline & 4 & 0 & 0 & 1 & 13 & \\
\hline \multirow{5}{*}{$\begin{array}{l}\text { Sites positive in ultrasono- } \\
\text { graphically }\end{array}$} & 0 & 0 & 6 & 0 & 0 & \multirow{3}{*}{$<0.001$} \\
\hline & 1 & 0 & 1 & 0 & 0 & \\
\hline & 2 & 0 & 6 & 6 & 0 & \\
\hline & 3 & 0 & 0 & 2 & 2 & \\
\hline & 4 & 0 & 0 & 5 & 11 & \\
\hline \multirow[t]{2}{*}{ Echotexture } & Hyperechoic & 0 & 10 & 13 & 13 & $<0.001$ \\
\hline & Hypoechoic & 40 & 3 & 0 & 0 & \\
\hline \multirow[t]{3}{*}{ Differentiation between layers } & Distinct & 0 & 0 & 0 & 0 & $<0.001$ \\
\hline & Normal & 40 & 10 & 0 & 0 & \\
\hline & Poor & 0 & 3 & 13 & 13 & \\
\hline \multirow[t]{3}{*}{ Submucosal thickness } & Increased & 0 & 4 & 13 & 13 & $<0.001$ \\
\hline & Mild increase & 0 & 7 & 0 & 0 & \\
\hline & Normal & 40 & 2 & 0 & 0 & \\
\hline \multirow[t]{2}{*}{ Vascularity } & Decreased & 0 & 0 & 8 & 13 & $<0.001$ \\
\hline & Normal & 40 & 13 & 5 & 0 & \\
\hline \multirow{2}{*}{ Gender } & Female & 6 & 4 & 2 & 1 & \multirow{2}{*}{0.489} \\
\hline & Male & 34 & 9 & 11 & 12 & \\
\hline
\end{tabular}


Graph 1. Comparison of thickness of submucosa of buccal mucosa between various groups and controls.

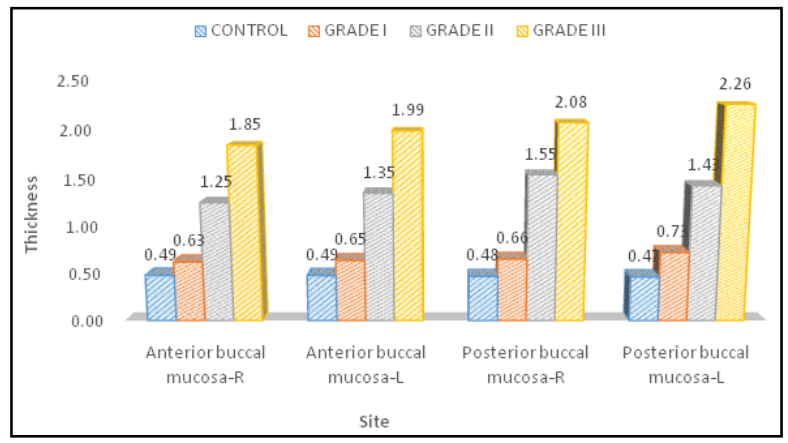

\section{Discussion}

OSMF is a chronic inflammatory potentially pre (malignant) disorder where the disease severity varies from site to site. It has a prevalence rate of 0.2 to $1.2 \%$ with a high malignant transformation rate of $3-7.6 \%$ and is very common in Indian population. It is incurable and irreversible even after the cessation of the habit $[3,9]$. It is characterized by progressive fibrosis and hence requires periodic monitoring, assessment of response to habit cessation and various medical and surgical treatment modalities. Multiple regions of oral cavity may be affected because OSMF is a diffuse disease. Hence even thoughbiopsy is considered as the final standard for the diagnosis of OSMF, it may not be possible to perform biopsy from multiple sites of the oral cavity for histopathological evaluation. And, biopsy taken from a single site may not represent the entire disease severity. Biopsy itself can induce scarring and further limit the mouth opening [2].

A Cochrane systematic review done on interventions for the management of OSMF pointed out that extraoral imaging may be used to assess the disease severity with high sensitivity [10]. Ultrasound is a safe, non-invasive, readily available, cost effective modality which provides real time imaging of the tissues being examined. Evidence for adverse biological effects of ultrasound used in diagnostic range is not reported in literature [11]. Evaluation of the tissues is done by assessing the difference in the thickness and echogenicity of the mucosal structures. It has also proved to be an efficient deep heating modality and if applied at higher intensity for a longer duration can soften the fibrous tissues [12]. It has been used for diagnosis and follow up of systemic diseases like scleroderma. Scleroderma is characterized by the presence of fibrosis and chronic inflammation like OSMF, but they differ in aetiology and clinical presentation which helps to differentiate them $[2,3]$. It was found out that areas with increased collagen deposition have high elastic moduli and present with hyperechogencity in ultrasound images [13]. Buccal mucosa is almost always involved in OSMF. Easy ultrasonographic examination is possible due to its superficial location and absence of underlying bone. Presence of fibrous bands in the buccal mucosa is its most common clinical presentation and in $4.5 \%$ of population, $90 \%$ of the epithelium overlying it becomes atrophic and it is the common site for malignant transformation [2].

As the tissue architecture will be relatively constant in the adult population, patients falling under the age group of 18-40 years was chosen for the study [2]. The age characteristics of the present study group were in accordance with the mean age of OSMF patients of 26.1 years as reported by the previous studies. The majority of the present cases were in the age range of $21-30$ years. Similar to the previously reported studies in this study also majority of the patients in the case group were men. In Indian society usage of commercial betel quid products is seen more among men than women and that may be the reason for male predominance in the present study also. This is alarming asit reflects the changing lifestyle of young Indian men [14].

Patients with systemic disease and other mucosal lesions which can result in fibrosis of the oral mucosa and alteration of vascularity were excluded from the study population as it can produce false positive results. Transcutaneous ultrasound examination was preferred as it will be difficult to place an intraoral transducer in patients with OSMF due to decreased interincisal mouth opening [2].

No OSMF cases which demonstrated palpable fibrous bands in clinical examination showed complete hypoechogenicity on US examination. This suggests that all cases of OSMF detected clinicallycan be also detected in ultrasound examination. It could even detect positive findings in stage 1 cases where there were no clinically palpable fibrous bands and hence it may be considered as an initial investigation modality in cases diagnosed with OSMF as the findings can affect the treatment protocol and prognosis of the patient. As the disease progresses from stage 1 to 3 submucosa could not be distinguished from the underlying muscle layer due to complete loss of echo differentiation between them. All these findings support the study results of Krithika et al who reports that complete loss of echo differentiation is highly indicative of well-established oral submucous fibrosis [2].

In the present study the mean submucosal thickness of controls was $0.48 \mathrm{~mm}$ which fell under the normal range between $0.45 \mathrm{~mm}$ $0.56 \mathrm{~mm}$. There was a steady increase in submucosal thickness from stage 1 to stage 3 cases in the case group. Rangaiah et al [3] also reported that submucosal thickness will be increased in OSMF patients, compared with controls which is similar to the present study. This is also in accordance to the study conducted by Agarwal et al, Tiwari et al and Devathambi and Aswath where they found statistical correlation between the severity of the disease and the submucosal thickness $[4,5,7]$. It was found that there was an increased thickness of submucosa in posterior buccal mucosa than the anterior buccal mucosa as most of the patients tends to keep the betel quid in that region. As disease progresses from stage 2 to stage 3 , significant increase in the submucosal thickness is noted. Nadendla et al in their study to assess the severity of OSMF by correlation between the clinical staging and sonographic findings found out that there is high correlation 
between the increase in submucosal thickness with the stage of the disease [11].

In our study we could find significant correlation between the OSMF stages and the findings from ultrasonography examination in all the groups except between the control and stage I group supporting the study done by Dupare et al [9].

Color Doppler examination can provide information about the vascularity which plays a significant role in the assessment of the outcome of the treatment modalities and prognosis of the disease condition. Decreased vascularity indicates poor prognosis, and more than average vascularity suggests malignant changes because of angiogenesis [9]. In the present study vascularity was found to be normal in stage 1 cases and is steadily decreasing as the disease severity increases. This supports the findings of study conducted by Manjunath et al who reports of decrease of vascularity in color Doppler ultrasound as the disease progresses [15]. Tiwari et al in a similar study reported of slight decrease in vascularity in stage I cases compared to control [16].

In the present study hyperechogenicity of the submucosa was present even in clinically negative sites on ultrasound examination. There was good correlation between the increase in submucosal thickness, loss of differentiation between the submucosa and muscle layer and increase in echogenicity of the mucosa with the stage of the disease. Hence Ultrasound Color Doppler examination can be considered as an adjunct to clinical examination in the assessment of the severity of OSMF and as an investigative modality especially in cases where it is not feasible to do a biopsy.

Histological grading is considered as the gold standard for executing the treatment plan of OSMF. In this study clinical staging of the disease was compared to the findings in the ultrasonographic examination, which is a potential limitation of the study. Future studies may be done evaluating the correlation between clinical and ultrasonography findings with histological grading.

\section{Acknowledgement}

We are very thankful to Dr. Rudresh Hiremath, Professor, Department of Radiology K V G Medical College and Hospital, Sullia and Dr. R Venkitachalam, Assistant Professor, Department of Public Health Dentistry Amrita School of Dentistry, Kochi for all the support and help provided.

\section{References}

[1]. van der Waal I. Historical perspective and nomenclature of potentially malignant or potentially premalignant oral epithelial lesions with emphasis on leukoplakia-some suggestions for modifications. Oral Surg Oral Med Oral Pathol Oral Radiol. 2018;125:577-81.

[2]. Krithika C, Ramanathan S, Koteeswaran D, Sridhar C, Satheesh J, Shiva Shankar MP. Ultrasonographic evaluation of oral submucous fibrosis in habitual areca nut chewers. Dentomaxillofac Radiol. 2013;42(9):1-8.

[3]. Rangaiah P, Annigeri RG, Lingappa A. Transcutaneous ultrasonographic assessment of oral submucous fibrosis: A preliminary study. Int J Oral Med Sci.2010;9:137-47.

[4]. Agarwal RK, Hebbale M, Mhapuskar A, Tepan M. Correlation of ultrasonographic measurements, histopathological grading, and clinical staging in oral submucous fibrosis. Indian J Dent Res. 2017;28:476-81.Pubmed PMID: 29072206.

[5]. Tiwari M, Gupta M, Ghom S, Devi B. Ultrasonographic evaluation of oral submucous fibrosis: A preliminary study. International Journal of Women Dentists 2014;1(1):1-4.Pubmed PMID: 24516775.

[6]. More CB, Das S, Adalja C, Kamatchi V, Venkatesh R. Proposed clinical classification for oral submucous fibrosis. Oral Oncol. 2012;48:200-2.Pubmed PMID: 22070918

[7]. Devathambi JR, Aswath N. Ultrasonographic evaluation of oral submucous fibrosis and masseteric hypertrophy. J Clin Imaging Sci. 2013;3 Suppl 1:12. Pubmed PMID: 24516775.

[8]. Bharat BM, Joshi JS, Patil SD. Triple Protocol Approach: A Modified Extended Approach of High Resolution Ultrasonography of cheek. Poster presented at: European Congress of Radiology 2011;Vienna, Australia.

[9]. Dupare A, Dhole A, Motwani M. Ultrasonographic evaluation of oral submucous fibrosis patients: A non-invasive diagnostic approach. J Indian Acad Oral Med Radiol. 2018;30:247-52.

[10]. Federowicz Z, Chan Shih-Yen E, Dorri M, Nasser M, Newton T, Shi L. Interventions for the management of oral submucous fibrosis. Cochrane Database Syst Rev .2009;1:CD007156.

[11]. Nadendla LK, Tatikonda VK, Bangi BB, Bhavya H, Devulapally RV, Pokala A. Sonographic imaging of fibrosis of oral mucosa and its correlation with clinical staging in oral submucous fibrosis. J Can Res Ther. 2018;14:394-7.

[12]. Dani VB, Patel SH. The effectiveness of therapeutic ultrasound in patients with oral submucosal fibrosis. Indian J Cancer 2018;55:248-50.Pubmed PMID: 30693888

[13]. Thapasum FA, Rangdhol V, Mohammed F, Mohamed S, Shanmugam S Gray-scale ultrasonographic imaging of the buccal mucosa in various stages of oral submucous fibrosis. Oral Radiol. 2014;31(3):143-8.

[14]. Reddy V, Wanjari PV, Bamda NR, Reddy P. Oral Submucous Fibrosis: Correlation of clinical grading to various habit factors. Int J Dent Clin. 2011;3:21-4.

[15]. Manjunath K, Rajaram PC, Saraswathi TR, Sivapathasundharam B, Sabarinath B, Koteeswaran D et al. Evaluation of oral submucous fibrosis using ultrasonographic technique: A new diagnostic tool. Indian J Dent Res. 2011;22:530-6

[16]. Tiwari M, Deoghare A, Sharma A, Saha S, Poptani R. Evaluation of OSMF with ultrasonography. Int J Oral Health Dent. 2017;3(3):169-74. 